\title{
Does lymphocyte-to-monocyte ratio before, during, or after definitive chemoradiation for locally advanced pancreatic cancer predict for clinical outcomes?
}

\author{
Irene Giacomelli ${ }^{1}$, Daniele Scartoni ${ }^{1}$, Homan Mohammadi ${ }^{2}$, William F. Regine ${ }^{3}$, Michael D. Chuong ${ }^{4}$ \\ ${ }^{1}$ Proton Treatment Center, APSS (Azienda Provinciale per i Servizi Sanitari), Trento, Italy; ${ }^{2}$ University of Maryland School of Medicine, Baltimore, \\ MD, USA; ${ }^{3}$ Radiation Oncology Department, University of Maryland, Baltimore MD, USA; ${ }^{4}$ Radiation Oncology Department, Miami Cancer \\ Institute at Baptist Health South Florida, Miami, Florida, USA \\ Contributions: (I) Conception and design: I Giacomelli, D Scartoni, MD Chuong; (II) Administrative support: MD Chuong, WF Regine; (III) \\ Provision of study materials or patients: MD Chuong, I Giacomelli, D Scartoni, WF Regine; (IV) Collection and assembly of data: I Giacomelli, D \\ Scartoni, H Mohammadi; (V) Data analysis and interpretation: I Giacomelli, D Scartoni, MD Chuong; (VI) Manuscript writing: All authors; (VII) \\ Final approval of manuscript: All authors. \\ Correspondence to: Irene Giacomelli, MD. Proton Treatment Center, APSS (Azienda Provinciale per i Servizi Sanitari), U.O. di Protonterapia, Via al \\ Desert, 1438123 Trento, Italy. Email: irenegiacomelli11@gmail.com.
}

Background: Elevated pre-treatment lymphocyte (L) to monocyte (M) ratio (LMR) in peripheral blood has been suggested to correlate with improved survival in some malignancies, but data in the context of pancreatic cancer (PC) is limited. The aim of this study was to evaluate the prognostic significance of LMR before, during and after definitive chemoradiotherapy (CRT) for locally advanced pancreatic cancer (LAPC).

Methods: We retrospectively reviewed 57 patients with LAPC treated with definitive CRT at a single institution from 2005 to 2013. Complete blood counts were obtained before (TP1), during the third week (TP2) and at the end of CRT (TP3). Univariate analysis (UVA) included gender, age, body mass index, pretreatment CA19-9, T stage, N stage, induction chemotherapy (ICT), absolute L count (TP1, TP2, TP3), absolute $\mathrm{M}$ count (TP1, TP2, TP3), LMR (TP1, TP2, TP3), and relative LMR changes (TP2 $\div$ TP1, TP $\div$ TP1, TP3 - TP2).

Results: Median follow-up was 14 months. Twelve patients received ICT. Median LMR was 2.7 (range, 0.8-5.25), 1.4 (range, 0.3-5) and 0.98 (range, 0.3-3.4) at TP1, TP2 and TP3, respectively. Superior PFS was significantly associated with an absolute $M$ count during CRT $<0.1(\mathrm{P}=0.04)$ while pre-CRT L count $\geq 1.1$ trended towards significance $(\mathrm{P}=0.09)$. Superior OS was significantly associated with change in LMR $(\mathrm{TP} 3 \div$ $\mathrm{TP} 2)>0.32(\mathrm{P}<0.0001)$ while pre-CRT LMR $\geq 2.6$ trended towards significance $(\mathrm{P}=0.06)$.

Conclusions: Factors significantly associated with overall survival (OS) and progression-free survival (PFS) were change in LMR at the end of CRT and absolute M count during CRT. This analysis suggests treatment-time-specific immune system parameters may affect clinical outcomes and warrant continued investigation.

Keywords: Pancreas; lymphocyte; monocyte; chemotherapy; radiotherapy

Submitted Mar 20, 2017. Accepted for publication May 18, 2017.

doi: 10.21037/jgo.2017.06.14

View this article at: http://dx.doi.org/10.21037/jgo.2017.06.14 


\section{Introduction}

Pancreatic cancer (PC) is the 4th most fatal cancer both in men and women and the 12th most common cancer in the United States. In 2017, it is estimated that more than 53,670 people will be diagnosed with PC (3.1\% of all new cancer cases) and 41,780 will die of this disease (7\% of all cancer deaths) (1). With a life expectancy of $\sim 5 \%$ at 5 years, the prognosis of this cancer has not significantly improved over the past 20 years, and incidence and mortality rates are very similar (2).

Treatment of patients with locally advanced pancreatic cancer (LAPC) remains highly controversial. Regardless of the treatment strategy, the average OS for these patients remains low $(<1$ year $)$ in the oldest studies, although it has marginally increased in recent trials $(3,4)$.

In the last years the attention of researchers has been focused in the inflammation as playing a major role in carcinogenesis. PC has been considered to be poorly immunogenic and thus not amenable to immunotherapy; this view has been challenged by several preclinical and clinical studies providing evidence that anti-tumor immune responses occur spontaneously and can be induced in patients with PC (5).

Immune system parameters have been proposed as prognostic biomarkers for patients with PC. Elevated pretreatment lymphocyte to monocyte ratio (LMR) in peripheral blood has been suggested to correlate with improved survival in some malignancies (6), but data in the context of PC is limited. Some studies analyzed the impact of pre-operative and post-operative LMR on OS in patients with PC, confirming the positive role of elevated pre-operative LMR and low levels of post-operative LMR on OS and prognosis $(7,8)$ The prognostic significance of LMR not only before, but also during and after definitive chemoradiotherapy (CRT) for locally advanced PC has not been previously reported and is the focus of this retrospective study.

\section{Methods}

\section{Patients}

We conducted a search of an IRB-approved database from the Department of Radiation Oncology at our institution to identify patients with LAPC who underwent definitive CRT for LAPC from December 2005 to September 2013. Inclusion criteria for the study included biopsy confirmation of adenocarcinoma of the pancreas, no evidence of distant metastasis, completion of definitive CRT and availability of complete blood cell counts (CBC) with differential before, during, and after CRT. LAPC was defined according to the National Comprehensive Cancer Network definitions (9), inclusive of superior mesenteric artery encasement greater than 180 degrees, any celiac abutment, inferior vena cava infiltration, unreconstructable superior mesenteric vein/ portal occlusion, and aortic invasion or encasement.

\section{Treatment characteristics}

All patients received definitive concomitant CRT with a median prescription dose of 50.4 Gy (range, 12-63 Gy) in a median of 28 fractions (range, 5-37). Intensity-modulated radiation therapy (IMRT) was primarily utilized; only 1 patient was treated with three-dimensional conformal radiation therapy.

Gross tumor volume (GTV) was defined using information obtained from endoscopic ultrasound, CT, and/or positron emission tomography. Clinical target volume (CTV) was generated using a $3-\mathrm{cm}$ superior and inferior and 1-cm axial expansion around the GTV and 1-cm isotropic expansion around grossly involved lymph nodes. For tumors located in the head of the pancreas, the elective nodal CTV was defined by the union of the lymphatic areas around the infrapyloric region, the common hepatic artery, the celiac trunk, the hepatoduodenal ligament, the posterior pancreaticoduodenal artery, the superior mesenteric artery, the paraaortic area and the anterior pancreaticoduodenal artery. In patients with pancreatic body-tail tumor lymph nodes around the common hepatic artery, the celiac trunk, the splenic artery and the hilum of spleen, the hepatoduodenal ligament, the superior mesenteric artery, and the paraaortic region. PTV was created using a $0.5-\mathrm{cm}$ expansion on the CTV using daily image guidance.

Concurrent chemotherapy was gemcitabine (27 patients), capecitabine (27 patients), 5 -FU (2 patients) and bevacizumab + capecitabine (1 patient). Twelve patients received also induction chemotherapy (ICT) with FOLFIRINOX (3 patients), gemcitabine ( 8 patients), gemcitabine + nab-paclitaxel (1 patient).

\section{LMR measurements}

CBC with differentials was recorded at specified time points (TP). The first (TP1) was after diagnosis and prior to start of CRT. The second (TP2) was during the third week of the CRT. The last (TP3) was at the end of CRT. Absolute monocyte and lymphocyte counts were recorded at each TP. The LMR at each TP was calculated by dividing the absolute 
Table 1 Patients and treatment characteristics

\begin{tabular}{|c|c|}
\hline Characteristic & $\begin{array}{l}\text { No. [\%] or } \\
\text { median (range) }\end{array}$ \\
\hline \multicolumn{2}{|l|}{ Sex } \\
\hline Male & $25[44]$ \\
\hline Female & $32[56]$ \\
\hline Median age (years) & 62 (range 38-84) \\
\hline \multicolumn{2}{|l|}{ Stage } \\
\hline I & $7[12]$ \\
\hline II & 20 [35] \\
\hline III & $30[53]$ \\
\hline \multicolumn{2}{|l|}{ Site } \\
\hline Head & $45[78]$ \\
\hline Body & $6[11]$ \\
\hline Periampullary-tail & $6[1]$ \\
\hline Median pre-treatment CA $19.9(\mathrm{U} / \mathrm{mL})$ & $\begin{array}{l}1,155 \\
\text { (range }<1-11,634)\end{array}$ \\
\hline Median radiotherapy dose (Gy) & 50.4 (range 12-64) \\
\hline \multicolumn{2}{|l|}{ Radiotherapy modality } \\
\hline IMRT & 56 [98] \\
\hline 3D CRT & $1[2]$ \\
\hline \multicolumn{2}{|l|}{ Induction chemotherapy } \\
\hline Folfirinox & $3[5]$ \\
\hline Gemcitabine & $8[14]$ \\
\hline Gemcitabine + Abraxane & $1[2]$ \\
\hline \multicolumn{2}{|l|}{ Concurrent chemotherapy } \\
\hline Gemcitabine & $27[47]$ \\
\hline Capecitabine or 5-FU & $29[51]$ \\
\hline Bevacizumab + Capecitabine & $1[2]$ \\
\hline
\end{tabular}

lymphocyte count by the absolute monocyte count. Absolute differences in LMR over time were calculated as follows: TP2 - TP1, TP3 - TP1, and TP3 - TP2. In addition to calculating absolute differences in LMR values, relative changes over time were calculated as follows: TP2 $\div$ TP1 (TP2/

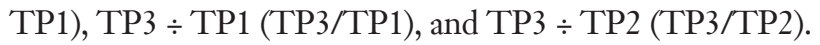

\section{Statistical analysis}

OS was defined as the interval from the date of diagnosis to date of death or last follow-up. Progression-free survival (PFS) was defined as the time interval from the date of diagnosis to date of distant or local recurrence. If no progression was noted, PFS was determined from the date of last follow-up or death. Univariate analysis (UVA) included gender, age, body mass index, pretreatment CA19-9, T stage, N stage, AJCC stage, ICT, absolute L count (TP1, TP2, TP3), absolute $M$ count (TP1, TP2, TP3), LMR (TP1, TP2, TP3), and relative LMR changes $(\mathrm{TP} 2 \div \mathrm{TP} 1, \mathrm{TP} 3 \div \mathrm{TP} 1, \mathrm{TP} 3 \div \mathrm{TP} 2)$. All factors significant on UVA with a $\mathrm{P}<0.15$ were evaluated in multivariate analyses. All statistical analyses were conducted using R software (10).

\section{Results}

A total of 57 patients were included in this analysis. The median age at diagnosis was 62 years. Thirty-two were female and 25 were male. Most had stage III (53\%) followed by stage II (35\%) disease, in according to the TNM classification (American Joint Committee on Cancer (AJCC) TNM staging system, 2013). In most cases the lesion was located on the head of the pancreas (72\%) followed by the body (10\%) (Table 1). The median pre-treatment CA 19.9 level was $1,155 \mathrm{U} / \mathrm{mL}$.

The median LMR at TP1 was 2.74 (range, 0.80-5.25); the median number of days between the TP1 lab values and start of definitive CRT was 3 days (range, $0-22$ days). The median LMR at TP2 was 1.4 (range, 0.3-7); the median number of days between the TP2 lab values and start of definitive CRT was 18 days (range, 7-48 days); the median LMR at TP3 was 0.98 (range, 0.13-3.40) and the median number of days between the TP3 lab values and the CRT completion was 7 days (range, 0-49 days).

With a median follow up of 14 months the median overall survival (OS) was 11 months. Superior PFS was significantly associated with an absolute $M$ count during CRT $<0.1(\mathrm{P}=0.04)$ while pre-CRT L count $\geq 1.1$ trended towards significance $(\mathrm{P}=0.07)$ on univariate and multivariate analysis (Table 2). Superior OS was significantly associated with a relative change in $\mathrm{LMR}(\mathrm{TP} 3 \div \mathrm{TP} 2)>0.32$ $(\mathrm{P}<0.0001)$ while pre-CRT $\mathrm{LMR} \geq 2.6$ trended towards significance $(\mathrm{P}=0.06)$ (Figures 1 and 2$)$.

\section{Discussion}

LMR as an inflammation and immunity-related biomarker based on lymphocytes and monocytes has been found to 
Table 2 At Cox regression analysis superior progression free survival (PFS) was significantly associated with an absolute Monocyte count during chemoradiotherapy $(C R T)<0.1(\mathrm{P}=0.04)$ while pre-CRT Lymphocyte count $\geq 1.1$ only trended towards significance $(\mathrm{P}=0.07)$ both on univariate and multivariate analysis. No other values in study were significantly associated with PFS

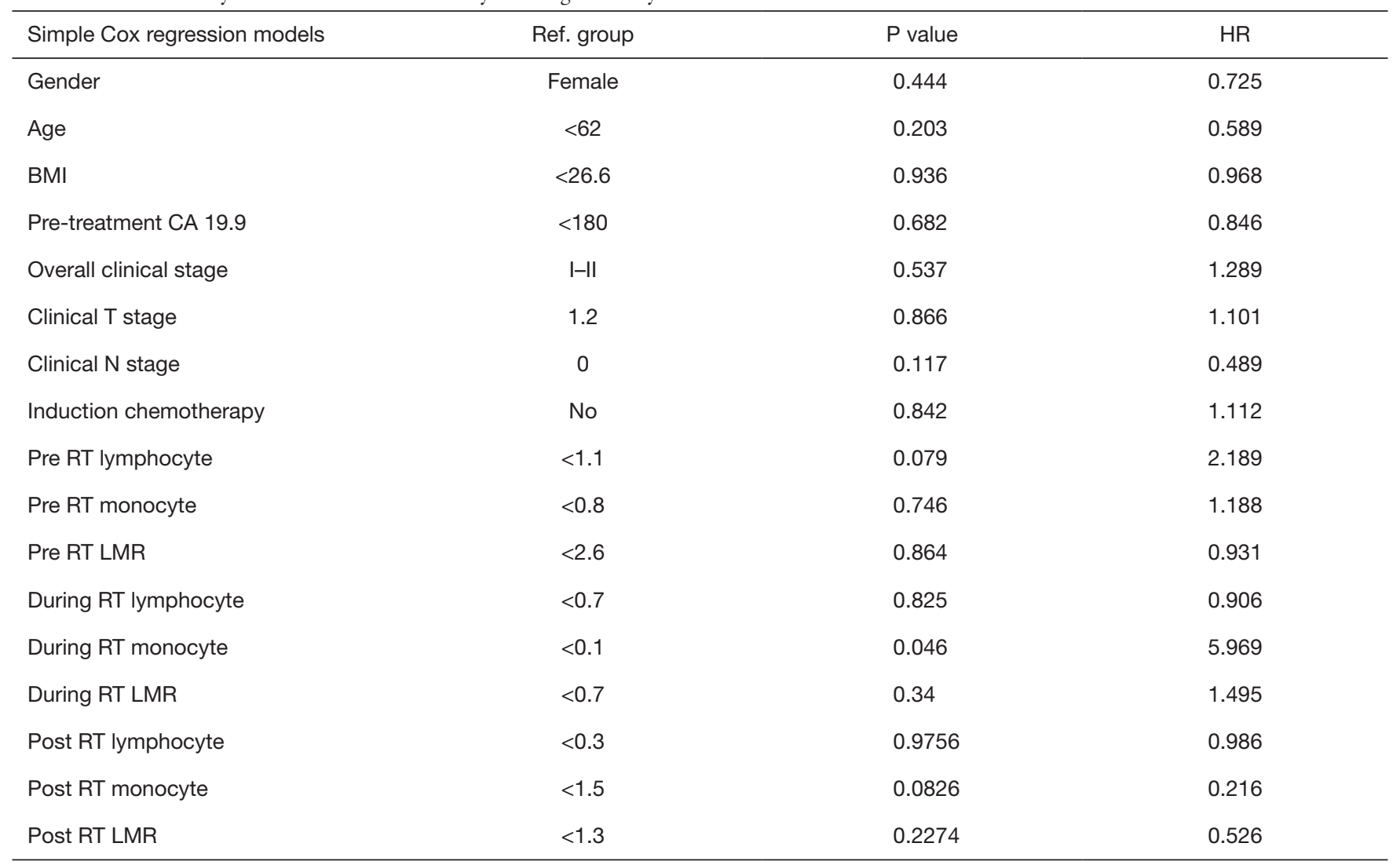

$\mathrm{RT}$, radiation therapy; LMR, lymphocyte to monocyte ratio; BMI, body mass index.

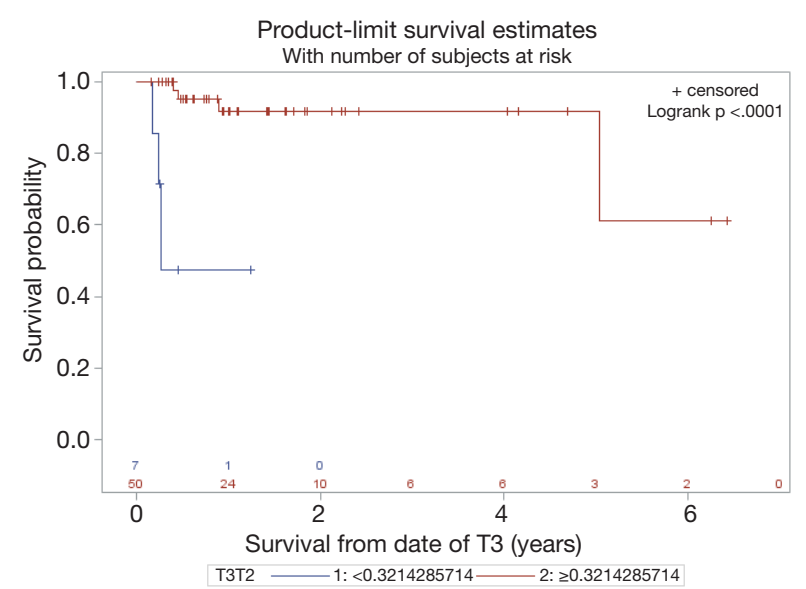

Figure 1 Superior pancreatic cancer overall survival (OS) was significantly associated with a change in lymphocyte to monocyte ratio (LMR) between third week of chemo-radiotherapy (CRT) (time point 2) and the end of CRT (time point 3) (time point $3 \div$ time point 2$)>0.32(\mathrm{P}<0.0001)$.

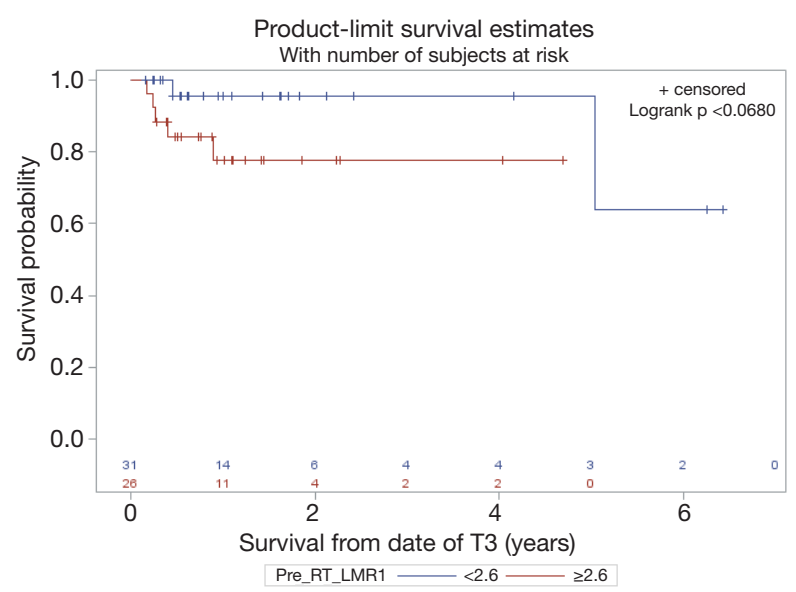

Figure 2 Pre-chemoradiotherapy lymphocyte to monocyte ratio (LMR) value $\geq 2.6$ trended towards significance $(\mathrm{P}=0.06)$ for overall survival (OS). 
be a significant prognostic marker for several malignancies (11-13). This ratio reflects both lymphopenia, resulting from weak, insufficient immunologic reaction to the tumor (14) and high levels of monocytes and macrophages present in the tumor microenvironment that support cancer cell invasion, migration, intravasation, angiogenesis and even lead to a suppression of anti-tumor immune reaction $(15,16)$. Stotz et al. analyzed 372 patients with stage II and III colon cancer and found that the LMR might be an independent prognostic marker for time to recurrence in stage III patients (12). Chen et al. evaluated the prognostic values of LMR in 485 patients with stage Ib1-IIa cervical cancer and found that decreased pretreatment LMR is associated with a poor prognosis in stage Ib1-IIa patients who underwent a radical operation (17).

A small number of studies analyzed the impact of LMR on OS and PFS in patients with pancreatic adenocarcinoma, most of which have studied the role of preoperative LMR as an independent prognostic factor for survival $(7,18)$. For example, Li et al. in his study analyzed a large cohort of patients who underwent curative resection for pancreatic adenocarcinoma and found a significant association of higher LMR with OS in univariate (19 vs. 12 months, $\mathrm{P}=0.000$ ), and multivariate analysis $(\mathrm{P}=0.000)$ and with median recurrencefree survival in univariate (18 vs. 10 months, $\mathrm{P}=0.000)$ and multivariate analyses (HR: $\mathrm{P}=0.000)$ (7).

To our knowledge our study is the first study to analyze the significance of LMR values at several TPs and their changes throughout treatment in pancreas cancer patients underwent definitive radio-chemotherapy. In our small sample size we demonstrate that factors significantly associated with OS and PFS were relative change in LMR $>0.32$ at the end of CRT and absolute monocyte count during CRT.

Radiation therapy and chemotherapy have wellknown adverse hematologic effects and, therefore, are expected to have a direct effect on LMR and on immune system in general. However, little data are available regarding these correlations, but it has been proven that radiation induces immunosuppression with the increase of immunosuppressive regulatory cells, direct bone marrow ablation, stimulation of inhibitory cytokines and direct destruction of mature circulating lymphocytes. Furthermore, peripheral lymphocytes are known to be extremely sensitive to radiation despite mitotic inactivity (19), however, the mechanism underlying the observed lymphocyte reduction during RT is unclear. With regard to lymphocyte effects on patient outcomes, clinical studies have shown an association between higher lymphocyte levels and better patient outcomes $(20,21)$. One of the largest of such studies was reported by Stanley et al. (22), who analyzed 77 variables in 5,000 Veterans Affairs patients with inoperable NSCLC. Among the variables analyzed, initial lymphocyte count ranked seventh in association with survival. These studies support our results where pre-CRT L count $\geq 1.1$ trended towards significance regarding PFS on Cox Regression model (Table 2). In addition, a recent study by Campian et al. (23) provides further support for the conclusions drawn from this study, correlating radiationinduced lymphopenia with significantly worse survival.

While we did not evaluate differences in radiation technique, the use of advanced radiation technologies including proton therapy may better spare bone marrow and the overall lymphocyte pool thereby improving the body's ability to mount a more effective anti-tumor response. Tang et al. (24) in a cohort of 711 patients who had received definitive radiotherapy for non-small cell lung cancer analyzed the correlation between GTVs, lung dose-volume histogram (DVH) parameters and lymphocyte nadirs with the results that larger GTV were correlated with lower lymphocyte and that patients with higher lymphocyte nadirs exhibited significantly improved OS. Analyses of lung DVH parameters revealed significant correlation at lower doses (lung V5-V10) that incrementally decreased and became no significant at higher doses (lung V60-V70).

Based on these studies and the results of our trial, it is evident how important is to preserve a high lymphocyte count and a high LMR during the CRT course. From the dosimetric point of view it would be interesting to analyze the correlation between DVH parameters and LMR in order to demonstrate, if it is possible, the presence of certain dosimetric values that impact on PFS and OS. The finding of dosimetric parameters associated with low LMR it may be the further incentive to perform a radiation modality treatment able to reduce the integral dose to the organs in which we assume a correlation with LMR.

\section{Conclusions}

Our study is the first to examine the significance of LMR at various TPs throughout CRT for locally advanced PC patients, showing that changes in LMR during treatment course are predictors of OS. Prospective evaluation is warranted to further elucidate the utility of such prognostic markers in PC. 


\section{Acknowledgements}

None.

\section{Footnote}

Conflicts of Interest: The authors have no conflicts of interest to declare.

Ethical Statement: It is a retrospective study and this study was approved by Institutional Review Board of University of Maryland (No. IRB00000233).

\section{References}

1. Howlader N, Noone AM, Krapcho M, et al. editors. SEER Cancer Statistics Review, 1975-2013, National Cancer Institute. Bethesda, MD.

2. Malvezzi M, Bertuccio P, Levi F et al. European cancer mortality predictions for the year 2014. Ann Oncol 2014;25:1650-6.

3. Hammel P, Huguet F, Van Laethem JL et al. Comparison of chemoradiotherapy (CRT) and chemotherapy (CT) in patients with a locally advanced pancreatic cancer (LAPC) controlled after 4 months of gemcitabine with or without erlotinib: Final results of the international phase III LAP 07 study. J Clin Oncol 2013;31:abstr LBA4003.

4. Loehrer PJ Sr, Feng Y, Cardenes H et al. Gemcitabine alone versus gemcitabine plus radiotherapy in patients with locally advanced pancreatic cancer: an Eastern Cooperative Oncology Group trial. J Clin Oncol 2011;29:4105-12.

5. Zheng L, Xue J, Jaffee EM, et al. Role of immune cells and immune-based therapies in pancreatitis and pancreatic ductal adenocarcinoma. Gastroenterology 2013;144:1230-40.

6. Gu L, Li H, Chen L, et al. Prognostic role of lymphocyte to monocyte ratio for patients with cancer: evidence from a systematic review and meta-analysis. Oncotarget 2016;7:31926-42.

7. Li GJ, Xu HW, Ji JJ, et al. Prognostic value of preoperative lymphocyte-to-monocyte ratio in pancreatic adenocarcinoma. Onco Targets Ther 2016;9:1085-92.

8. Fujiwara Y, Misawa T, Shiba H, et al. Postoperative peripheral absolute blood lymphocyte-to-monocyte ratio predicts therapeutic outcome after pancreatic resection in patients with pancreatic adenocarcinoma. Anticancer Res 2014;34:5163-8.

9. National Comprehensive Cancer Network. NCCN
Clinical Practice Guidelines in Oncology, Version 1.2014. Available online: http://www.nccn.org//professionals/ physician_gls/pdf/pancreatic.pdf, accessed 10 Feb 2014.

10. R Core Team. A language and environment for statistical computing. R Foundation for Statistical Computing, Vienna, Austria. Available online: http://www.R-project.org

11. Zhang GM, Zhu Y, Luo L, et al. Preoperative lymphocytemonocyte and platelet-lymphocyte ratios as predictors of overall survival in patients with bladder cancer undergoing radical cystectomy. Tumour Biol 2015;36:8537-43.

12. Stotz M, Pichler M, Absenger G, et al. The preoperative lymphocyte to monocyte ratio predicts clinical outcome in patients with stage III colon cancer. Br J Cancer 2014;110:435-40.

13. Zhou X, Du Y, Xu J, et al. The preoperative lymphocyte to monocyte ratio predicts clinical outcomes in patients with stage II/III gastric cancer. Tumour Biol 2014;35:1165966.

14. Hoffmann TK, Dworacki G, Gooding W, et al. Spontaneous apoptosis of circulat- ing T lymphocytes in patients with head and neck cancer and its clinical importance. Clin Cancer Res 2002;8:2553-62.

15. Pollard JW. Tumour-educated macrophages promote tumour progression and metastasis. Nat Rev Cancer 2004;4:71-8.

16. Condeelis J, Pollard JW. Macrophages: obligate partners for tumor cell migration, invasion, and metastasis. Cell 2006;124:263-6.

17. Chen L, Zhang F, Sheng XG, et al. Decreased pretreatment lymphocyte/monocyte ratio is associated with poor prognosis in stage Ib1-IIa cervical cancer patients who undergo radical surgery. Onco Targets Ther 2015;8:1355-62.

18. Stotz M, Szkandera J, Stojakovic T, et al. The lymphocyte to monocyte ratio in peripheral blood represents a novel prognostic marker in patients with pancreatic cancer. Clin Chem Lab Med 2015;53:499-506.

19. Sellins KS, Cohen JJ. Gene induction by gammairradiation leads to DNA fragmentation in lymphocytes. J Immunol 1987;139:3199-206.

20. Leibowitz-Amit R, Israel A, Gal M, et al. Association between the Absolute Baseline Lymphocyte Count and Response to Neoadjuvant Platinum-based Chemotherapy in Muscle-invasive Bladder Cancer. Clin Oncol (R Coll Radiol) 2016;S0936-6555:30185-6.

21. Jang JE, Kim YR, Kim SJ, et al. A new prognostic model using absolute lymphocyte count in patients with primary central nervous system lymphoma. Eur J Cancer 
2016;57:127-35.

22. Stanley KE. Prognostic factors for survival in patients with inoperable lung cancer. J Natl Cancer Inst 1980;65:25-32.

23. Campian JL, Ye X, Brock M, et al. Treatment-related lymphopenia in patients with stage III non-small-cell lung

Cite this article as: Giacomelli I, Scartoni D, Mohammadi H, Regine WF, Chuong MD. Does lymphocyte-to-monocyte ratio before, during, or after definitive chemoradiation for locally advanced pancreatic cancer predict for clinical outcomes? J Gastrointest Oncol 2017;8(4):721-727. doi: 10.21037/ jgo.2017.06.14 cancer. Cancer Invest 2013;31:183-8.

24. Tang C, Liao Z, Gomez D, et al. Lymphopenia association with gross tumor volume and lung V5 and its effects on non-small cell lung cancer patient outcomes. Int J Radiat Oncol Biol Phys 2014;89:1084-91. 